Bauwelt Fundamente 4

Herausgegeben von Peter Neitzke

Beirat: Gerd Albers Hildegard Barz-Malfatti Elisabeth Blum Eduard Führ Thomas Sieverts Jörn Walter 



\section{Jane Jacobs}

\section{Tod und Leben großer amerikanischer Städte}

Mit einem Nachwort von Gerd Albers

Bauverlag

Gütersloh · Berlin
Birkhäuser

Basel 
Originaltitel The Death and Life of Great American Cities (Random House, 1961)

Übersetzung der vorliegenden, gekürzten Ausgabe von Eva Gärtner

Die Reihe Bauwelt Fundamente wurde von Ulrich Conrads 1963 gegründet und bis 2013 herausgegeben (einschließlich Band 149), seit Anfang der 1980er Jahre gemeinsam mit Peter Neitzke.

Library of Congress Cataloging-in-Publication data

A CIP catalog record for this book has been applied for at the Library of Congress.

Bibliografische Information der Deutschen Nationalbibliothek

Die Deutsche Nationalbibliothek verzeichnet diese Publikation in der Deutschen Nationalbibliografie; detaillierte bibliografische Daten sind im Internet über http://dnb.dnb.de abrufbar.

Dieses Werk ist urheberrechtlich geschützt. Die dadurch begründeten Rechte, insbesondere die der Übersetzung, des Nachdrucks, des Vortrags, der Entnahme von Abbildungen und Tabellen, der Funksendung, der Mikroverfilmung oder der Vervielfältigung auf anderen Wegen und der Speicherung in Datenverarbeitungsanlagen, bleiben, auch bei nur auszugsweiser Verwertung, vorbehalten. Eine Vervielfältigung dieses Werkes oder von Teilen dieses Werkes ist auch im Einzelfall nur in den Grenzen der gesetzlichen Bestimmungen des Urheberrechtsgesetzes in der jeweils geltenden Fassung zulässig. Sie ist grundsätzlich vergütungspflichtig. Zuwiderhandlungen unterliegen den Strafbestimmungen des Urheberrechts.

Dieses Buch ist auch als E-Book (ISBN 978-3-0356-o212-8) erschienen.

Der Vertrieb über den Buchhandel erfolgt ausschließlich über den Birkhäuser Verlag.

(C) 2015 Birkhäuser Verlag GmbH, Basel, Postfach 44, 4.009 Basel, Schweiz, ein Unternehmen von Walter de Gruyter GmbH, Berlin/Boston;

und Bauverlag BV GmbH, Gütersloh, Berlin

\section{bau $\|$ | verlag}

Gedruckt auf säurefreiem Papier, hergestellt aus chlorfrei gebleichtem Zellstoff. TCF $\infty$

Printed in Germany

ISBN $97^{8-3-7643-635^{6-7}}$

987654321

www.birkhauser.com 
NEW YORK CITY gewidmet. Wohin ich kam auf der Suche nach dem Glück, und wo ich es fand in Bob, Jimmy, Ned und Mary auch für sie ist dieses Buch geschrieben.

Es haben mir so viele Menschen bei diesem Buch bewußt und unbewußt geholfen, daß ich niemals in der Lage sein werde, mich für die ganze Verpflichtung, die ich schulde und empfinde, erkenntlich zu zeigen. Im besonderen danke ich den folgenden Personen dafür, daß sie mir mit Informationen, Hilfe oder Kritik zur Seite gestanden haben:

Saul Alinsky, Norris C. Andrews, Edmund Bacon, June Blythe, John Decker Butzner jr., Henry Churchill, Grady Clay, William C. Crow, Vernon de Mars, Monsignor John J. Egan, Charles Farnsley, Carl Feiss, Robert B. Filley, Mrs. Rosario Folino, Chadbourne Gilpatric, Viktor Gruen, Frank Havey, Goldie Hoffman, Frank Hotchkiss, Leticia Kent, William H. Kirk, Mr. und Mrs. George Kostritsky, Jay Landesman, Rev. Wilbur C. Leach, Glennie M. Lenear, Melvin F. Levine, Edward Logue, Ellen Lurie, Elizabeth Manson, Rober Montgomery, Richard Nelson, Joseph Passonneau, Ellen Perry, Rose Porter, Ansel Robison, James W. Rouse, Samuel A. Spiegel, Stanley B. Tankel, Jack Solkman, Robert C. Weinberg, Erik Wensberg, Henry Whitney, William H. Whyte jr., William Wilcox, Mildred Zucker, Bede Zwicker. Selbstverständlich ist niemand von ihnen verantwortlich für das, was ich geschrieben habe; einige sind ausgesprochen gegenteiliger Ansicht und haben mir trotzdem in großzügiger Weise geholfen.

Ich bin außerdem zu Dank verpflichtet der Rockefeller Foundation für die finanzielle Unterstüztung, die meine Arbeit und dieses Buch ermöglichte, ferner der New School for Social Research für ihre Gastfreundschaft, und schließlich Douglas Haskell, dem Herausgeber des Architectural Forum, für seine Ermutigungen und für seine Geduld. Den größten Dank schulde ich jedoch meinem Mann, Robert H. Jacobs jr.; allmählich weiß ich nicht mehr, welche Gedanken dieses Buches seine und welche meine sind.

JANE JACOBS 
*Bis vor kurzem war das Beste, was die Zivilisation mir außer der blinden Hinnahme einer Ordnung des Universums bedeutete, daß sie den Künstler, den Dichter, den Philosophen und den Wissenschaftler möglich macht.

Ich denke jetzt, daß das nicht das Größte ist. Jetzt glaube ich, daß das Größte etwas ist, was uns alle direkt angeht.

Wenn man sagt, daß wir uns zu sehr mit den Umständen des Lebens beschäftigen, dann ist meine Antwort, daß der Hauptwert der Zivilisation gerade darin liegt, $d a \beta$ sie die Lebensbedingungen komplexer macht; daß sie große und zusammenhängende geistige Bemühungen von uns verlangt an Stelle von einfachen und zusammenhanglosen, damit die Menschheit ernährt und gekleidet wird und Wohnungen erhält und von einem Ort zum anderen befördert wird. Weil komplexere und intensivere geistige Bemühungen ein volleres und reicheres Leben bedeuten, bedeuten sie mehr Leben überhaupt. Leben ist ein Ziel in sich, und die Frage, ob es lebenswert ist, ist die Frage, ob man genug von ihm hat.

Nur ein Wort noch.

Wir sind alle der Verzweiflung sehr nahe.

Die Planken, die uns über die Wellen der Verzweiflung hinwegtragen, bestehen aus Hoffnung, aus dem Glauben an den unerklärlichen Wert und unbezweifelbaren Sinn der Bemühung und aus der tiefen, unterbewußten Befriedigung, welche uns die Ausübung unserer Fähigkeiten schenkt.* OLIVER WENDELL HOLMES ir. 